\title{
SIMULATION OF GROUND MOTION INDUCED PROTON BEAM ORBIT VIBRATION IN HERA
}

\section{Montag, DESY, Hamburg, Germany*}

\section{Abstract}

A ground motion model based on realistic spectra and correlation properties has been developed in order to simulate ground motion induced beam orbit vibration. This model has been used to simulate the motion of the HERA proton beam. Simulation results and comparisons with orbit measurements will be presented.

\section{INTRODUCTION}

Ground motion induced beam jitter plays an important role for many kinds of accelerators, like future linear colliders, synchrotron radiation sources or storage rings. The resulting beam orbit vibration potentially leads to luminosity degradation in colliding beam facilities due to beam offset at the interaction point and/or emittance growth.

The sensitivity of beam orbits to quadrupole motion depends strongly on the correlation length of ground motion at a specific frequency. Quadrupole motion with a correlation length exceeding the betatron wavelength has only little influence on beam jitter [1]. Therefore any simulation of ground motion effects has to take into account not only the power spectrum of motion at a single point but also the coherence properties of ground motion.

This paper presents a ground motion simulation model which has been originally developed for linear accelerators [2]. It is based on measured power spectra and coherence properties of ground motion. This model is extended to circular machines in order to simulate ground motion induced beam jitter in the HERA proton ring.

\section{THE SIMULATION MODEL}

As measurements at different geographical locations show, the power spectrum $P_{\text {tot }}(\omega)$ of ground motion at a single point can be roughly approximated as [3]

$$
P_{\text {tot }}(\omega)=\frac{B}{\omega^{4}},
$$

where $B$ is some proportionality constant characteristic of the site, and $\omega=2 \pi f$. According to the $A T L$ rule [4], the power spectrum $\rho(\omega, L)$ of the uncorrelated motion of two points at a distance $L$ is [3]

$$
\rho(\omega, L)=\frac{A \cdot L}{\omega^{2}} .
$$

Obviously, the uncorrelated part of motion must be smaller than the total motion, thus

$$
\rho(\omega, L) \leq P_{\text {tot }}(\omega) \text { for all } \omega .
$$

\footnotetext{
*Email: christoph.montag@ desy.de
}

According to Equations (1) and (2), this is violated for frequencies $\omega>\sqrt{(B /(A \cdot L))}$. Therefore Equation (2) can only be valid for frequencies below this limit, while it has to be modified in the frequency domain above. This is achieved by a redefinition of $\rho(\omega, L)$ as

$$
\rho(\omega, L)=\left\{\begin{array}{l}
\frac{A \cdot L}{\omega^{2}}, \omega<\sqrt{B /(A \cdot L)} \\
\frac{B}{\omega^{4}}, \omega \geq \sqrt{B /(A \cdot L)}
\end{array}\right.
$$

For the description of transverse motion of $N$ quadrupoles in a circular accelerator, we start with $N+1$ independent time series $x_{n}(t)$, each of them having the same power spectrum $P_{n}=P_{\text {tot }}=B / \omega^{4}$.

The coherent part of the motion of the $(n+1)$ st magnet with respect to the $n$th one can be calculated by applying a first order lowpass filter with cutoff frequency $\omega_{0}=\sqrt{B /(A \cdot L)}$ to the motion $y_{n}(t)$ of the $n$th magnet. The resulting power spectrum of this correlated motion in terms of the Laplace variable $s=i \omega$ is

$$
\begin{aligned}
P_{\text {corr }, n+1}(\omega) & =\left|\frac{\omega_{0}}{s+\omega_{0}}\right|^{2} \cdot P_{\text {tot }, n}(\omega) \\
& =\left|\frac{\omega_{0}}{s+\omega_{0}}\right|^{2} \cdot \frac{B}{\omega^{4}} .
\end{aligned}
$$

The corresponding uncorrelated part of motion of the $(n+$ 1)st magnet with respect to the $n$th one is calculated applying a first order high pass filter with the same cutoff frequency $\omega_{0}$ to an independent motion signal $x_{n+1}(t)$ with power spectrum $P_{n+1}$, resulting in

$$
P_{\text {uncorr }, n+1}(\omega)=\left|\frac{s}{s+\omega_{0}}\right|^{2} \cdot P_{n+1}(\omega) .
$$

In the low frequency limit, this spectrum reflects the $A T L$ rule:

$$
\lim _{\omega \rightarrow 0} P_{\text {uncorr }, n+1}=\frac{A \cdot L_{n+1}}{\omega^{2}},
$$

where $L_{n+1}$ is the distance between the $n$th and the $(n+$ 1)st magnet.

As can be shown, $P_{\text {corr }, n+1}+P_{\text {uncorr }, n+1}=P_{n+1}=$ $P_{\text {tot }}=B / \omega^{4}$, while the resulting coherence $\left|\gamma_{n+1}\right|$ is calculated as [2]

$$
\begin{aligned}
\left|\gamma_{n+1}\right| & =\sqrt{\frac{P_{\text {corr }, n+1}}{P_{\text {uncorr }, n+1}} /\left(1+\frac{P_{\text {corr }, n+1}}{P_{\text {uncor }, n+1}}\right)} \\
& =\sqrt{\frac{\omega_{0}^{2}}{|s|^{2}+\omega_{0}^{2}}} \\
& =\sqrt{\frac{B /(A \cdot L)}{|s|^{2}+B /(A \cdot L)}} .
\end{aligned}
$$


This scheme is repeated for all $N$ magnets, starting with $y_{1}(t)=x_{1}(t)$. To reflect the periodicity of the circular accelerator, the motion $y_{N+1}(t)$ of an $(N+1)$ st magnet is formally calculated from the motion $y_{N}(t)$ of the $N$ th magnet by application of the corresponding filters, with $L_{N+1}$ being the distance between the $N$ th and the 1st magnet. Obviously, the motion of the $(N+1)$ st magnet has to be identically the same as the motion of the 1 st magnet. Therefore all magnet positions $y_{n}(t)$ are corrected according to

$$
y_{n}^{*}(t)=y_{n}(t)-\frac{y_{N+1}(t)-y_{1}(t)}{L_{\mathrm{tot}, N+1}} \cdot L_{\mathrm{tot}, n} .
$$

Here $L_{\text {tot }, n}=\sum_{k=2}^{n} L_{k}$ is the total distance between the 1 st and the $n$th magnet, i. e. $L_{\mathrm{tot}, N+1}$ is the circumference of the machine.

To get a more realistic model, the experimentally obtained ground motion data in the HERA tunnel at DESY are fitted as [2]

$$
\begin{aligned}
P_{\text {tot }}= & \left|\frac{B}{\omega^{4}}\right| \\
& +\left|\frac{4.225 \cdot 10^{-14}}{s^{2}}\right| \mathrm{m}^{2} \cdot \mathrm{Hz} \\
& +\left|\frac{7 \cdot 10^{-6}}{s^{2}+9.425 \mathrm{~Hz} \cdot s+246.74 \mathrm{~Hz}^{2}}\right|^{2} \mathrm{~m}^{2} \mathrm{~Hz}^{3} \\
& +\left|\frac{7 \cdot 10^{-7}}{s^{2}+0.528 \mathrm{~Hz} \cdot s+0.774 \mathrm{~Hz}^{2}}\right|^{2} \mathrm{~m}^{2} \mathrm{~Hz}^{3} .
\end{aligned}
$$

In the low frequency limit this spectrum is dominated by the $B / \omega^{4}$ term, thus leading to an $A T L$-like behaviour of the uncorrelated motion. The second term represents some additional cultural noise at high frequency, while the third and the fourth term reflect some $2.5 \mathrm{~Hz}$ resonance of the upper earth's crust and the microseismic peak at $1 / 7 \mathrm{~Hz}$, respectively.

\section{RESULTS}

The ground motion model described in the previous section has been applied to the vertical motion of the HERA proton ring, using $A=4 \cdot 10^{-6} \mu \mathrm{m}^{2} /(\mathrm{m} \cdot \mathrm{sec})$ as obtained on the DESY site [5]. The parameter $B$ is chosen at $B=3 \cdot 10^{-2} \mu \mathrm{m}^{2} \cdot \mathrm{Hz}^{3}$, resulting in good agreement of the power spectrum (Eq. (10)) as well as the coherence (Eq. (8)) with measured data.

Starting with a perfectly aligned machine, the orbit change is obtained by tracking a single particle, with initial conditions being on the design orbit, while the transverse quadrupole positions $y^{*}(t)$ are changed from turn to turn. For this tracking, only linear elements are taken into account. The resulting beam motion is monitored at a single point in such a way that the motion of this "monitor" itself is subtracted. This motion signal is normalized to a $\beta$-function of $\beta_{\text {mon }}=1 \mathrm{~m}$. Figure 1 shows the resulting power spectrum, which has been averaged over several samples. As a comparison with measured data (Fig. 2)

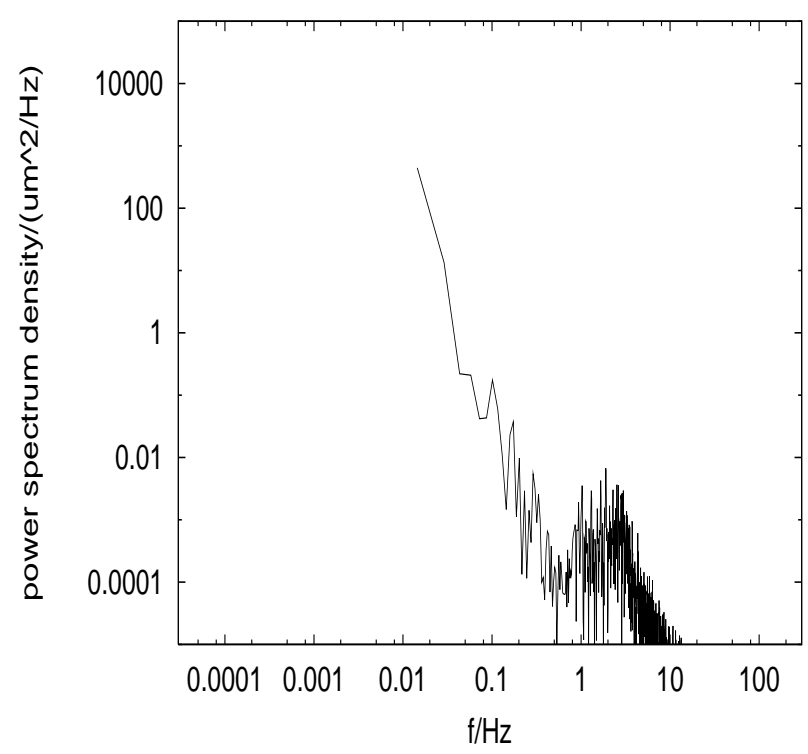

Figure 1: Power spectrum of simulated vertical beam jitter in HERA-p, normalized to $\beta_{\text {mon }}=1 \mathrm{~m}$.

shows, the simulated spectrum reflects the general properties of observed beam motion. The enhancement of orbit vibration amplitudes around $2.5 \mathrm{~Hz}$ is clearly seen in both spectra, but the frequency region in which this enhancement occurs stretches to higher frequencies (up to $50 \mathrm{~Hz}$ ) in the measured beam motion. This can possibly be explained by mechanical resonances of magnet supports which are not reflected by the ground motion spectrum used for simulation. The same argument also holds for the clearly visible $50 \mathrm{~Hz}$ peak caused by either mechanical vibration of some electric equipment or by direct noise on the cables.

In both spectra the microseismic peak at $1 / 7 \mathrm{~Hz}$ almost vanishes due to the large correlation length of this lowfrequency motion.

Quantitatively, both power spectra agree within a factor of 10 , which corresponds to a factor of $\sqrt{10}$ in amplitude. This difference can possibly be explained by the uncertainties in the parameters $A$ and $B$.

\section{CONCLUSION}

As has been shown, simulated ground motion induced beam orbit vibration amplitudes in the circular accelerator HERA agree within a factor of about 3 with measured data. For a more realistic simulation, mechanical resonances of the magnet supports, for example, should be taken into account in order to achieve a better agreement in the frequency region above a few Hertz.

\section{ACKNOWLEDGEMENTS}

The author is indebted to B. Holzer for many helpful discussions, and to M. Minty for reading the manuscript. 
Proceedings of the 1999 Particle Accelerator Conference, New York, 1999

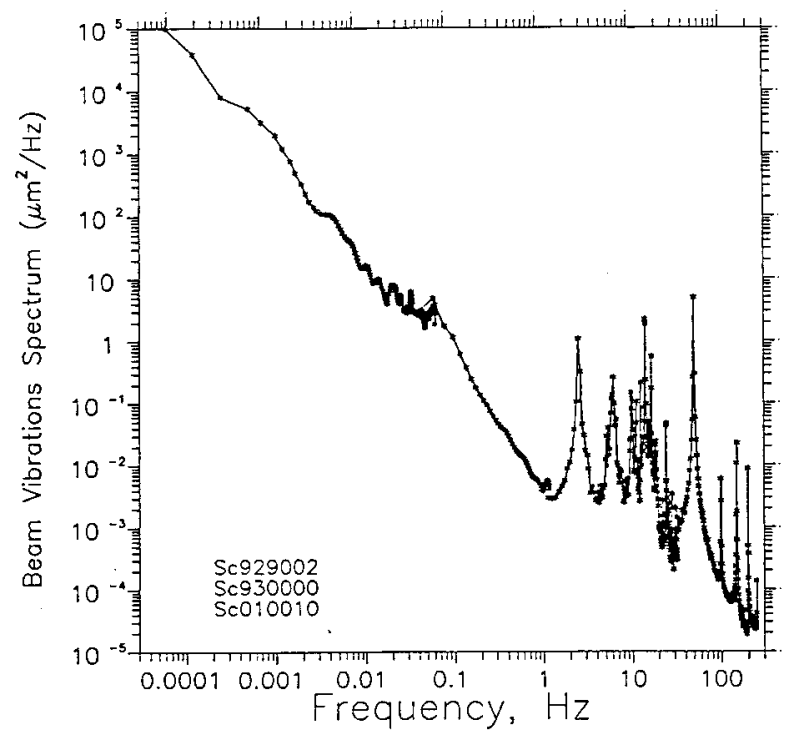

Figure 2: Measured power spectrum of vertical beam motion in HERA-p, normalized to $\beta_{\mathrm{mon}}=1 \mathrm{~m}$ [6].

\section{REFERENCES}

[1] J. Rossbach, Closed-Orbit Distortions of Periodic FODO Lattices Due to Plane Ground Waves, Part. Acc. 23 (1988) pp. 121-132

[2] C. Montag, Active Stabilization of Mechanical Quadrupole Vibrations in a Linear Collider Test Facility, DESY 97-030

[3] A. Sery, O. Napoly, Influence of Ground Motion on the Time Evolution of Beams in Linear Colliders, Phys. Rev. E 63 (1996)

[4] B. Baklakov et al., Investigation of Seismic Vibrations for Linear Collider VLEPP, Sov. Phys. ZhTF., Vol. 63, No. 10 (1993) (in Russian)

[5] R. Brinkmann, J. Rossbach, Observation of Closed Orbit Drift at HERA Covering 8 Decades of Frequency, Nucl. Instr. Meth. A 350 (1994)

[6] V.Shiltsev, B. Baklakov, P.Lebedev, C. Montag, J. Rossbach, Measurements of Ground Vibrations and Orbit Motion at HERA, DESY HERA 95-06 